\title{
COMPETITIVE ADSORPTION OF BIMODAL LATEX SUSPENSION*
}

\author{
Jakub Barbasz ${ }^{\mathrm{a}, \mathrm{b} \dagger}$, Michą Cieśla ${ }^{\mathrm{b} \ddagger}$, Aneta Michna ${ }^{\mathrm{a}}$ \\ ${ }^{a}$ Jerzy Haber Institute of Catalysis and Surface Chemistry \\ Polish Academy of Sciences \\ Niezapominajek 8, 30-239 Kraków, Poland \\ ${ }^{\mathrm{b}}$ The M. Smoluchowski Institute of Physics, Jagiellonian University \\ Reymonta 4, 30-059 Kraków, Poland
}

(Received May 27, 2013)

Competitive deposition of binary mixture of small and large latex particles is studied using Random Sequential Adsorption (RSA) algorithm. We designated the saturated random coverage ratio dependence on small-tolarge particle concentration ratio and on particles size ratio. Moreover, the deposition kinetics of the process was calculated numerically. To check validity of the numerical model, the saturated random coverage ratio for 1:1 binary latex particle mixture was measured experimentally using Scanning Electron Microscopy (SEM) and Atomic Force Microscopy (AFM).

DOI:10.5506/APhysPolB.44.945

PACS numbers: 05.45.Df, $68.43 . \mathrm{Fg}$

\section{Introduction}

Irreversible adsorption plays an important role in bio, material and food sciences [1, 2], and also in pharmaceutical [3] and cosmetic industries [4]. For example, it has been proved that thrombosis formation on cardiovascular and other implants is induced by protein deposition [5]. Moreover, protein adsorption processes play an important role also in blood coagulation, artificial organ failure, plaque formation, fouling of contact lenses [6]. Controlled particles adsorption is a prerequisite for efficient separation and

* Presented at the XXV Marian Smoluchowski Symposium on Statistical Physics, "Fluctuation Relations in Nonequilibrium Regime", Kraków, Poland, September 10-13, 2012.

$\dagger$ ncbarbas@cyf-kr.edu.pl

$\ddagger$ michal.ciesla@uj.edu.pl

$\S$ ncmichna@cyf-kr.edu.pl 
purification by chromatography and filtration [7]. Because of its fundamental and practical significance, numerous studies have been carried out over last few decades with the aim of evaluating deposition kinetics and properties of the obtained mono- and multilayers [8-10].

Theoretical modelling of adsorption processes started in 1980, when Feder introduced Random Sequential Adsorption (RSA) algorithm used for simulation of spheres deposition on a flat, homogeneous, two dimensional surface [11]. Since then, most of scientific effort was focused on modelling adsorption of monodisperse particles like spheres [12], spheroids [13] and spherocylinders [14] as well as more complex particles like dimers [15], polymers [16] or even proteins like fibrinogens [17, 18]. In most of these topics, RSA appeared to be successful in explaining fundamental properties of monolayers, such as maximal coverage ratio and monolayer structure. Adsorption of polydisperse macromolecules have also been a subject of numerous studies, however, typically a continuous distribution of particles size was assumed $[19,20]$. On the other hand, there are many processes, where adsorbed particles can have only several, specified dimensions e.g. proteins solutions [17, 21, 22] and, therefore, their size distribution cannot be approximated by a continuous function.

This study is focused on the simplest case of such adsorption, where spherical particle size distribution is bimodal. Latest experimental effort in this field, e.g. [23-26], the raises a question about accuracy of theoretical models of irreversible deposition. Therefore, the main aim of present work is to check if the saturated random coverages ratio obtained numerically using RSA algorithm agrees well with an experimental data. Similar theoretical approach has been used by Subashiev et al. [27], who however, have only analysed deposition on a one dimensional collector.

\section{Model}

We assumed that there are two types of spherical particles in a solution. The only difference between them is their sizes. The concentration of particles are $c_{\mathrm{S}}$ and $c_{\mathrm{L}}$ for the small and the large ones, respectively. When a particle touches the collector surface, it is irreversibly adsorbed. Theoretical model of such adsorption bases on Random Sequential Adsorption (RSA) algorithm [11]. The method iteratively adds individual particle to an adsorption film as follows:

(i) a new virtual spherical particle is randomly created. Its centre is set on the collector according to a uniform probability distribution. Its size is chosen randomly with probability $q$ for small and $1-q$ for large particle, where

$$
q=\frac{c_{\mathrm{S}}}{c_{\mathrm{S}}+c_{\mathrm{L}}}
$$


(ii) the virtual particle undergoes overlapping test with its nearest neighbours; if there is no overlap, the virtual particle is added to the existing layer;

(iii) otherwise the virtual particle is removed and abandoned.

The numerical experiments were done using squared $20 \mu \mathrm{m}$ collector. Each simulation lasted $10^{5} S_{\mathrm{C}} / S_{\mathrm{S}}$ time steps, where $S_{\mathrm{C}}=400 \mu \mathrm{m}^{2}$ is the collector area and $S_{\mathrm{S}}=\pi\left(d_{\mathrm{S}}^{2}\right) / 4 \mu \mathrm{m}^{2}$ is the area covered by smaller particle. The diameters of particles used during simulation were $d_{\mathrm{S}}=142 \mathrm{~nm}$ and $d_{\mathrm{L}}=780 \mathrm{~nm}$. The simulation was performed for $q \in[0.1,0.9]$. For each specified set of parameters, at least 10 independent numerical experiments were performed to get enough statistics for further data analysis.

The adsorption layer is mainly characterised by a total coverage ratio $\theta$. It denotes the part of a collector area occupied by adsorbed particles. In the case of our simulation, it is a sum of coverages given by small and large particles

$$
\theta=\theta_{\mathrm{L}}+\theta_{\mathrm{S}}
$$

where

$$
\theta_{\mathrm{L}}=n_{\mathrm{L}} \frac{\pi d_{\mathrm{L}}^{2}}{S_{\mathrm{C}}}, \quad \theta_{\mathrm{S}}=n_{\mathrm{S}} \frac{\pi d_{\mathrm{S}}^{2}}{S_{\mathrm{C}}},
$$

and where $n_{\mathrm{L}}$ and $n_{\mathrm{S}}$ are numbers of large and small adsorbed particles, respectively. The coverage ratio rises during simulation and formally, after an infinite number of steps, it approaches the saturated random coverage ratio $\theta_{\max }$. Practically, after $10^{5} S_{\mathrm{C}} / S_{\mathrm{S}}$ steps, there were almost no depositions, and, therefore in this study, $\theta_{\max }$ denotes coverage ratio after that simulation time.

\section{Numerical results}

Typical adsorption layers obtained from simulation were shown in Fig. 1. It is worth to notice that the obtained concentration on a collector surface is significantly different from the value $q$ describing particle solution over a collector.
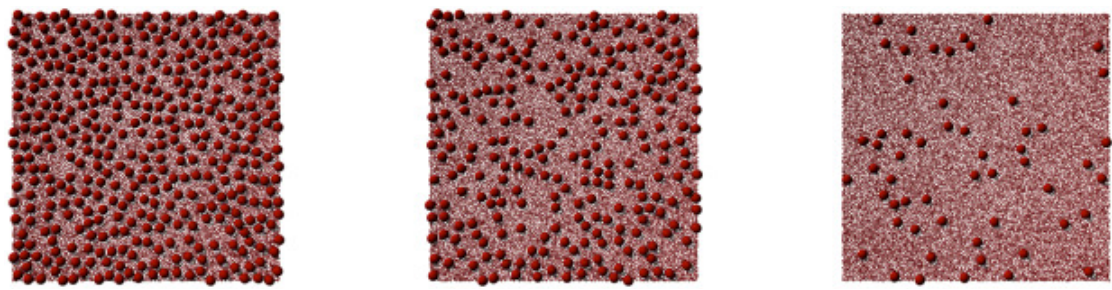

Fig. 1. Saturated coverages obtained from RSA simulations for three different concentration ratios: $q=0.1$ (left), $q=0.5$ (centre) and $q=0.9$ (right). 


\subsection{Saturated random coverage ratio}

The dependence of the saturated random coverage ratio on $q$, for both types of particles, is drawn in Fig. 2. When $q$ is large (much more smaller particles), the coverage given by small spheres tends to a $\theta_{\mathrm{S}, \max }=0.53$, which is very close to a well-known value of the maximal random coverage for adsorbate containing spherical particles: 0.54 [11]. Similarly, when $q$ tends to 0, large balls fill the collector surface with the similar ratio. However, if there are any small balls, they will fill remaining uncovered space. In a limit of $d_{\mathrm{L}} \gg d_{\mathrm{S}}$, this additional coverage could theoretically rise up to $\theta_{\mathrm{S}, \max }=(1-0.54) \times 0.54 \approx 0.25$, so the total coverage ratio will approach $\theta_{\max }=0.79$. Here, when the particle radius ratio is approximately 0.18 , the total coverage at $q \rightarrow 0$ rises up to $\theta_{\max }=0.76$.

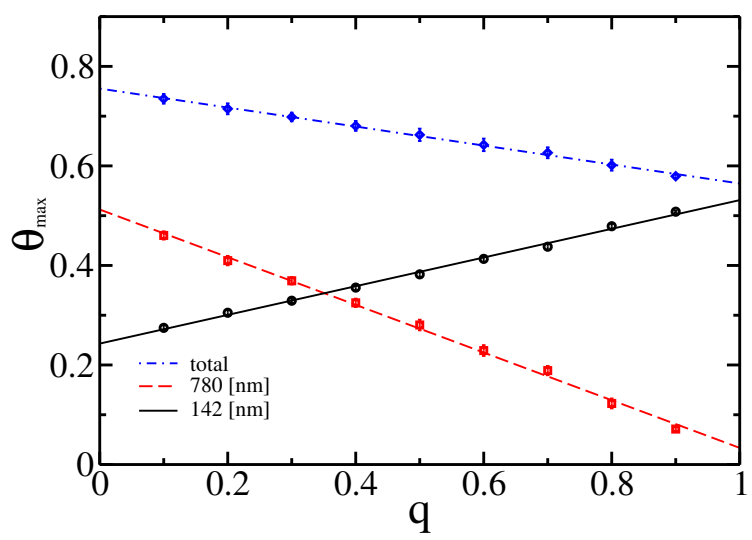

Fig. 2. Participation in the total coverage $\theta_{\max }$ of both types of spheres. Presented fits are: $\theta_{\max }(q)=-0.191 q+0.775$ and $\theta_{\mathrm{L}, \max }(q)=-0.479 q+0.512$ for large particles, and $\theta_{\mathrm{S}, \max }(q)=0.288 q+0.243$ for small ones.

What is even more interesting, the above relations can be well approximated by linear fits. For example, the coverage share of large particles is approximated by: $\theta_{\mathrm{L}, \max }=-0.48 q+0.51$. Here, due to quite strong dependence of $\theta_{\mathrm{S} \text {,max }}$ on $q$, this specific fit can be particularly useful for precise estimating concentration of small particles in a solution by measuring only the large ones, which have been adsorbed. The linear dependence of the $\theta_{\max }$ on $q$ has previously been observed and analytically supported only for one dimensional competitive bimodal adsorption [27].

The above results were obtained for specific particle radius ratio: $\left(d_{\mathrm{S}}=\right.$ $142 \mathrm{~nm}) /\left(d_{\mathrm{L}}=780 \mathrm{~nm}\right)=0.182$. The plot presented in Fig. 3 answers the question how the saturated coverage depends on particle size ratio. Here, the concentration ratio in a solution was fixed at $q=0.5$. Interestingly, the dependence is weak. We noticed that it can be well approximated by 
a quadratic function in a wide range of size ratio, but such approximation clearly fails near $d_{\mathrm{S}} / d_{\mathrm{L}}=1$, where both, large and small coverages have to meet at $\theta_{\mathrm{L}, \max }=\theta_{\mathrm{S}, \max }=0.54 / 2=0.27$.

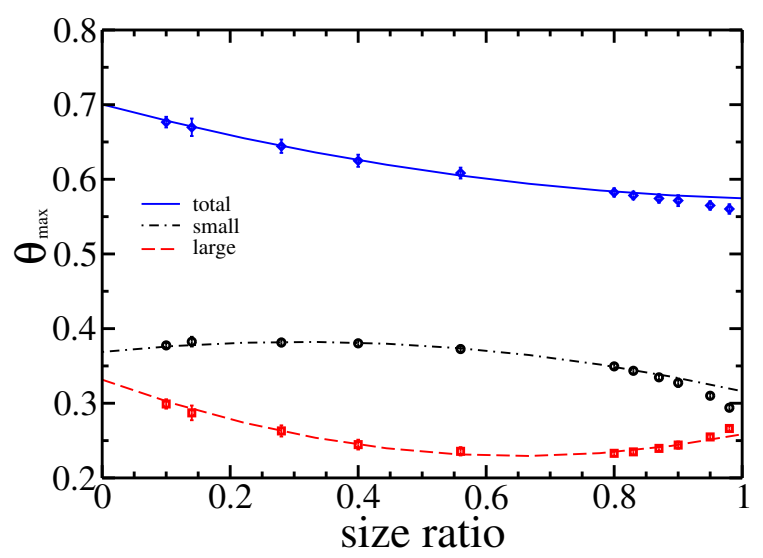

Fig. 3. Dependence of the saturated random coverage ratio on particles diameters ratio $d_{\mathrm{S}} / d_{\mathrm{L}}$ for $q=0.5$. Lines are square functions fitted to the numerical data.

\subsection{Adsorption kinetics}

Another area of comparison between simulations and experiments is adsorption kinetics, namely the growth of the adsorption monolayer. Unfortunately, the direct comparison cannot be done, as there is no physical time in RSA scheme. The kinetic of the physical process is governed not only by an deposition probability but also by a transport mechanism, which brings particles to the collector surface. Therefore, the reaction speed depends mainly on two factors: the number of particles close to the surface and ready to be adsorbed, and the probability of finding enough free space for successful deposition. The first factor can be estimated by modelling transport processes involved into an experiment - typically diffusion or flow. The second one can be calculated directly from an RSA simulation as a ratio of successful to all tries when adding a particle to existing coverage. This probability is also known as an Available Surface Function (ASF) and depends on a coverage ratio $\theta$. Figure 4 shows the ASF for large and small particles separately.

The plot shows different character of adsorption for both types of balls. The large ones adsorb faster at least in a sense of space they are blocking, however, when the total coverage exceeds $\theta_{\max } / 2$, there are practically no more places for them on a collector. The ASF for small particles indicates slower, linear decay at the beginning. Due to significant size difference, there is no additional excluded surface other than the one previously covered by 


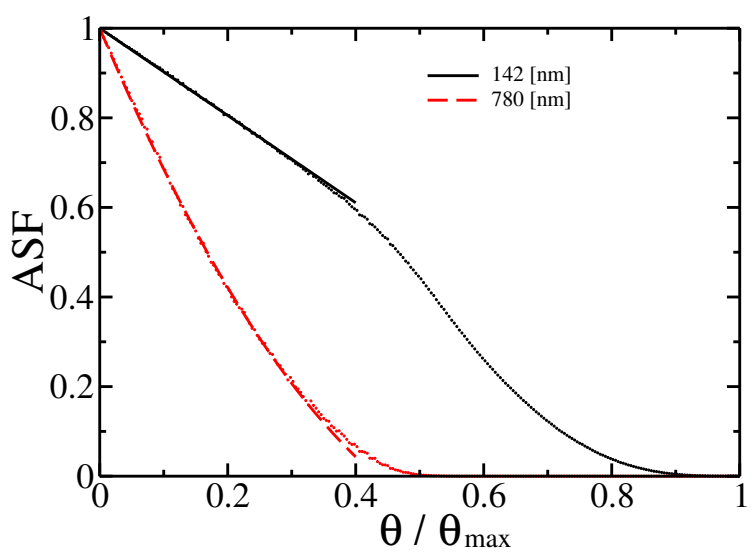

Fig. 4. ASF function for $142 \mathrm{~nm}$ and $780 \mathrm{~nm}$ balls for $q=0.5$. Fitted functions for small coverages are: $1-3.397\left(\theta / \theta_{\max }\right)+2.513\left(\theta / \theta_{\max }\right)^{2}$ for large and $1-0.974\left(\theta / \theta_{\max }\right)$ for small particles.

large balls. When $\theta>\theta_{\max } / 2$, the coverage ratio rises only because of small particle adsorption. Further rapid drop of ASF reflects the lack of the uncovered space, which is blocked also by smaller particles.

Assuming the transport process provides constantly the same relative particle concentration near surface as in a solution, the kinetics could be examined by the dependence of $\theta_{\mathrm{L}}$ on $\theta_{\mathrm{S}}$, which should be easily measured in experiment. Results of numerical simulation are presented in Fig. 5. The $\theta_{\mathrm{L}}$ saturates faster for small values of the ratio $q$, which is consistent with conclusions driven from ASF behaviour. To get dependence between coverage ratio and physical time, the specific transport mechanism of deposited par-

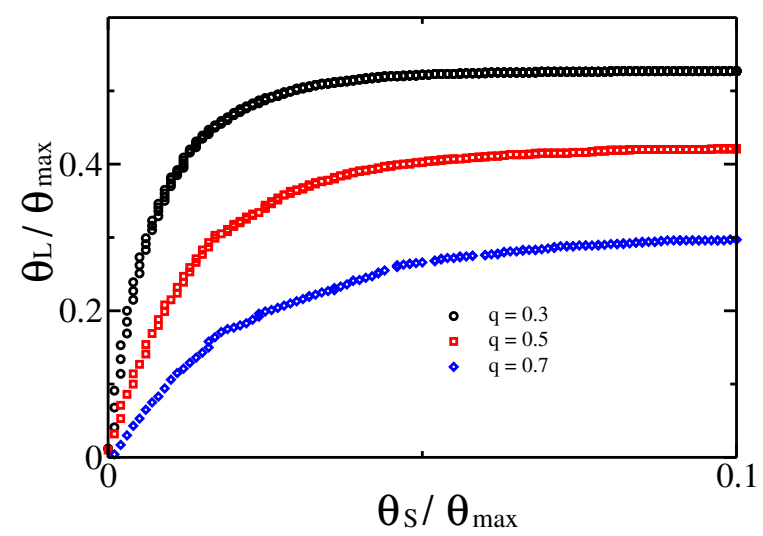

Fig. 5. Dependence of $\theta_{\mathrm{L}} / \theta_{\max }$ on $\theta_{\mathrm{S}} / \theta_{\max }$ for three different values of $q$. 
ticles should be taken into account. For example, the calculation scheme for diffusion driven adsorption is presented in details in [28] and for convection in [21].

\section{Experimental results}

Numerical results presented in Sec. 3 were compared with experimental data obtained by analysing adsorption of a binary mixture of latex particles on mica surface.

\subsection{Materials}

All materials used in the experiment were analytic reagents and were used without prior purification. The sodium chloride, sodium hydroxide and the hydrochloric acid were purchased from POCH. Ultrapure water was obtained using the Milli-Q Elix \& Simplicity purification system from Millipore SA Molsheim, France. Natural ruby mica sheets supplied by Continental Trade Ltd., Poland were used as the substrate surfaces for latex deposition experiments. Thin sheets were freshly cleaved and used in each experiment without any pretreatment.

The positively charged latex suspensions were produced in a surfactantfree polymerisation procedure with the special azonitrile indicator [12]. In this study, two types of monodisperse latex samples were applied. Both synthesised suspensions were characterised in the bulk by measuring their diffusion coefficients $D$ in ionic strength $I=10^{-3} \mathrm{M}$ and $\mathrm{pH}=5.5$ by applying the Dynamic Light Scattering method (DLS). The averaged size (hydrodynamic diameter) of particles was $d_{\mathrm{L}}=774 \pm 41 \mathrm{~nm}$ (larger particles) and $d_{\mathrm{S}}=145 \pm 3 \mathrm{~nm}$ (smaller particles). Therefore, their size ratio $d_{\mathrm{S}} / d_{\mathrm{L}}$ equal to 0.187 was almost the same as in RSA modelling.

\subsection{Experimental procedure}

The concentrations of particles in both monodisperse suspensions $c_{\mathrm{S}}$ and $c_{\mathrm{L}}$ were determined by using densitometer [29]. In a separate series of experiments, the parallel depositions of both suspensions were performed. In order to do this, the freshly cleaved mica sheets were immersed either into monomodal suspension containing one type of particles (774 nm or $145 \mathrm{~nm}$ ) or into the bimodal mixture. In all cases, the bulk concentration of particles in colloidal suspension was $8.4 \times 10^{9} \mathrm{~cm}^{-3}$. Therefore, the concentration ratio $q$ was either 0 (only larger particles), 1 (only smaller particles) or 0.5 (mixture both types of particles where $c_{\mathrm{L}}=c_{\mathrm{S}}$ ).

The mica sheets, submersed in such mixtures, were manipulated with stainless-steel forceps to prevent contamination and were held vertically in the suspension to avoid deposition of aggregates by sedimentation. Particles 
were deposited for a fixed time, ranging from 5 minutes to 10 days. Substrates were then washed by ultrapure water to remove any non-adsorbed nanoparticles in the fluid film, and air-dried prior to imaging. Finally, the samples were analysed using SEM technique. SEM measurements were conducted using the JEOL JSM-7500F Field Emission instrument at $15 \mathrm{kV}$. Furthermore, the samples were imaged using AFM technique. Adsorption experiments were conducted under the diffusion controlled transport.

\subsection{Saturated random coverage ratio}

AFM micrographs of exemplary monolayers formed after $t=24 \mathrm{~h}$ for monodispersive substrates are shown in Fig. 6. The structure of covering layer is significantly different than the one obtained numerically and presented in Fig. 1. The strong aggregation, especially of large particles, is caused by drying of the sample, which is necessary for AFM and SEM measurements. Despite of changes of the structure of adsorbed layers, the drying does not affect the coverage ratio [30]. In both measured cases, the coverage ratios is $\theta=0.47$ and the statistical error of measurements is below $10 \%$.
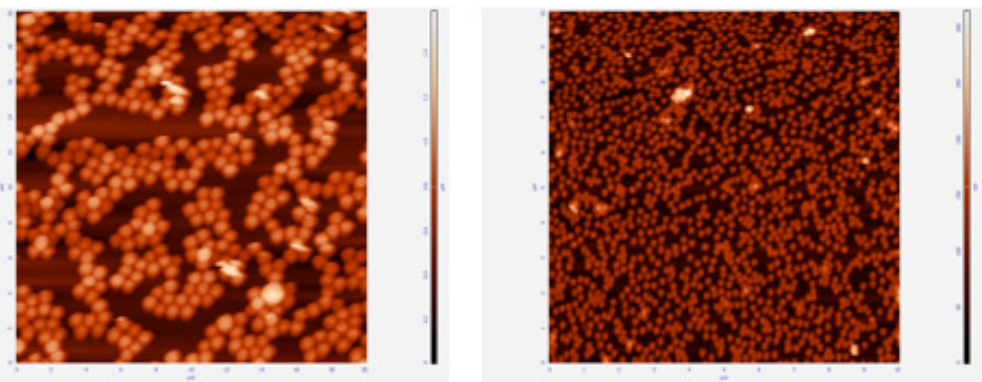

Fig. 6. AFM micrographs of large (left) and small (right) latex particles on mica. Deposition conditions: bulk suspension concentration $c=8.4 \times 10^{9} \mathrm{~cm}^{-3}, t=24 \mathrm{~h}$, $\mathrm{pH}=5.5, I=10^{-3} \mathrm{M}$. The scale bars are: $20 \mu \mathrm{m}$ (left) and $10 \mu \mathrm{m}$ (right).

The saturated coverages for mixed substrates were determined experimentally for $q=0.5$. The ionic strength was equal to $I=10^{-3} \mathrm{M}, \mathrm{pH}=5.5$. Experiments lasted for $t=70 \mathrm{~h}$ and then the mica surface was imaged by scanning microscopy. The typical structure formed by large and small particles is presented in Fig. 7. It was found that in these conditions the saturated coverage ratio of large particles was equal to $\theta_{\mathrm{L}, \max }=0.28$. This value correlates quite well with the theoretically calculated maximum surface coverage $\theta_{\mathrm{L}, \max }=0.27$. However, the coverage ratio of small latexes was found equal to $\theta_{\mathrm{S}, \max }=0.06$, whereas the theoretically calculated value was $\theta_{\mathrm{S}, \max }=0.39$. This discrepancy between theory and experiment can be, at least partially, explained by covering small particles by the large ones. 


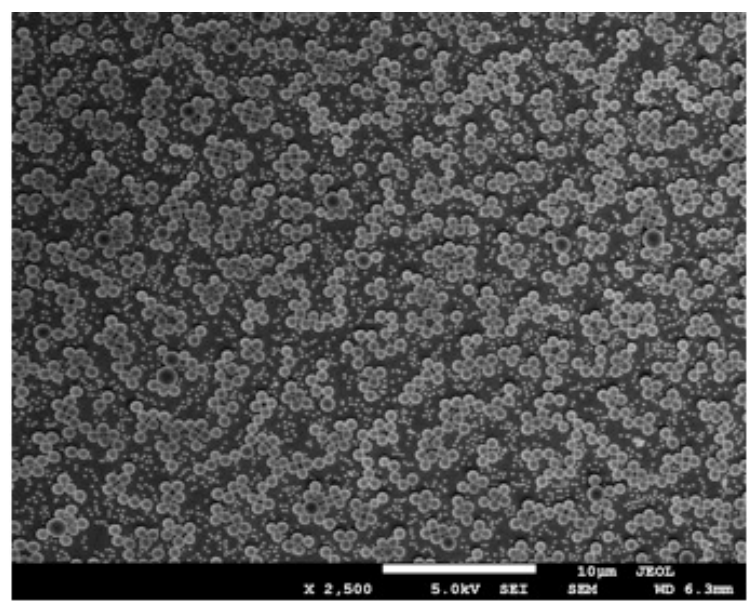

Fig. 7. SEM image of latex particles layer on mica obtained by an adsorption from bimodal suspension. Deposition conditions: bulk suspension concentrations of both latexes $c_{\mathrm{S}}=c_{\mathrm{L}}=8.4 \times 10^{9} \mathrm{~cm}^{-3}, t=70 \mathrm{~h}, \mathrm{pH}=5.5, I=10^{-3} \mathrm{M}$. The image size $47.47 \times 35.65 \mu \mathrm{m}^{2}$.

\section{Conclusions}

The concentration ratio in experiment can be determined, in convenient way, by density measurements of appropriate supernatant solution and colloidal suspension. The appropriate sizes of large and small particles in $10^{-3} \mathrm{M}$, pH 5.5 were successfully determined by the Dynamic Light Scattering method.

Coverages of large particles obtained numerically by the RSA algorithm agree with experimental data at least for concentration ratio of $q=0.0$ and $q=0.5$. Coverages of small particles are harder to measure experimentally and, therefore, their comparison cannot be done here. Moreover, numerical modelling shows that the saturated coverage ratio obtained during competitive irreversible adsorption of bimodal latex particles strongly depends on small to large particle concentration ratio. Its dependence on particle size ratio is weaker.

Due to experimental techniques used here, this study cannot answer the question if the RSA modelling reproduces correctly the structure of monolayers obtained experimentally.

This work was supported by the Polish Ministry of Science and Higher Education grant N N204 439040. 


\section{REFERENCES}

[1] M. Adamczak et al., Colloids Surf. B90, 211 (2011).

[2] J. Petlicki, T.G.M. van de Ven, Colloids Surf. A83, 9 (1994).

[3] A. Rafati et al., J. Contr. Rel. 162, 321 (2012).

[4] V.K. Gupta, A. Mittal, L. Kurup, J. Mittal, J. Colloid Interf. Sci. 304, 52 (2006).

[5] C.A. Haynes, W. Norde, Colloids Surf. B2, 517 (1994).

[6] B. Hall, L. Jones, J.A. Forrest, J. Biol. Med. Mater. Res. A 101A, 755 (2013).

[7] T. Rimmele, J.A. Kellum, Critical Care 15, 205 (2011).

[8] Z. Adamczyk et al., Adv. Colloid Interf. Sci. 153, 1 (2010).

[9] M. Rabe, D. Verdes, S. Seeger, Adv. Colloid Interf. Sci. 162, 87 (2011).

[10] T. Wei, S. Kaewtathip, K. Shing, J. Phys. Chem. C113, 2053 (2009).

[11] J. Feder, J. Theor. Biol. 87, 237 (1980).

[12] Z. Adamczyk, B. Siwek, M. Zembala, P. Belouschek, Adv. Colloid Interf. Sci. 48, 151 (1994).

[13] Z. Adamczyk, P. Weronski, Langmuir 11, 4400 (1995).

[14] P. Viot, G. Tarjus, S.M. Ricci, J. Talbot, J. Chem. Phys. 97, 5212 (1992).

[15] M. Ciesla, J. Barbasz, J. Stat. Mech. 03, P03015 (2012).

[16] M. Ciesla, Phys. Rev. E87, 052401 (2013).

[17] Z. Adamczyk, J. Barbasz, M. Ciesla, Langmuir 27, 6868 (2011).

[18] A. Bratek-Skicki, P. Zeliszewska, Z. Adamczyk, M. Ciesla, Langmuir 29, 3700 (2013).

[19] Z. Adamczyk, B. Siwek, M. Zembala, P. Weronski, J. Colloid Interf. Sci. 185, 236 (1997).

[20] P. Hanarp, D.S. Sutherland, J. Gold, B. Kasemo, J. Colloid Interf. Sci. 241, 26 (2001).

[21] Z. Adamczyk, J. Barbasz, M. Ciesla, Langmuir 26, 11934 (2010).

[22] M. Panos, T.Z. Sen, M.G. Ahunbay, Langmuir 28, 12619 (2012).

[23] N. Giamblanco et al., Soft Matter 8, 8370 (2012).

[24] M. Ombelli et al., Biofouling: J. Bioadhesion Biofilm Res. 27, 505 (2011).

[25] S.A. Maurer, C.N. Bedbrook, C.J. Radke, Langmuir 28, 14598 (2012).

[26] J. Wei et al., Biomed. Mater. 4, 045002 (2009).

[27] A.V. Subashiev, S. Luryi, Phys. Rev. E76, 011128 (2007).

[28] M. Ciesla, J. Barbasz, Surf. Sci. 612, 24 (2013).

[29] A. Michna, Z. Adamczyk, M. Ocwieja, E. Bielanska, Colloids Surf. A377, 261 (2011).

[30] M. Nattich-Rak et al., Colloids Surf. A412, 72 (2012). 